\title{
Metodología para evaluación de la condición ambiental en microcuencas urbanas
}

\section{Methodology for assessment of the environmental condition in urban waters microbasins}

\author{
Oscar Guarín-Villamizar ${ }^{1}$ \\ ${ }^{1}$ Grupo de Desarrollo Experimental y Tecnológico-GEDETEC, Tecnología en Supervisión de Obras Civiles, Universidad de \\ Santander, Colombia. Email: oguarin@udes.edu.co
}

RECIBIDO: Mayo 29, 2016. ACEPTADO: Marzo 11, 2017. VERSIÓN FinAL: Mayo 17, 2017.

\begin{abstract}
RESUMEN
Esta investigación se concentra en la presentación de una metodología para la rehabilitación productiva y el manejo sostenible de cualquier microcuenca urbana, sin embargo, para efectos prácticos, se centra en la quebrada "La Calavera" del municipio de Floridablanca Colombia, el fin es disminuir la contaminación a través de un exhaustivo análisis físico, químico y microbiológico seguido del aprovechamiento del espacio público proponiendo la recuperación medioambiental de microcuencas urbanas, con un enfoque eco turístico resaltando la flora y fauna, que enmarca este tipo de paisajes, con una topografía propicia para el deleite y la práctica de deportes en espacios naturales. Este diagnóstico ambiental permite el planteamiento de soluciones eficaces y oportunas para proteger la salud y mejorar la calidad de vida no solo de los habitantes de los barrios aledaños a la microcuenca sino a comunidades de características similares.
\end{abstract}

PALABRAS CLAVE: Microcuenca, Evaluación, Diagnóstico, Metodología.

\begin{abstract}
This investigation centers in the presentation of a methodology for the productive rehabilitation and the sustainable managing of any urban microbasin, nevertheless, for practical effects, centres on the gully " The Skull " of Floridablanca Colombia's municipality which end is to diminish his pollution across an exhaustive physical, chemical and microbiological analysis followed by the utilization of the public space proposing the alternative for the environmental recovery of urban microbasins, with an approach tourist echo highlighting the flora and fauna, which frames this type of landscapes, with a propitious topography for the delight and the practice of sports in natural spaces. This environmental diagnosis allows the exposition of solution effective and opportune to protect the health and to improve the quality of life not only of the inhabitants of the bordering neighborhoods to the microbasin but to communities of similar characteristics.
\end{abstract}

KEYWORDS: Microbasin, Evaluation, Diagnostic, Methodology.

\section{INTRODUCCIÓN}

El desarrollo urbanístico de las ciudades de forma descontrolada ha contribuido infortunadamente a la depredación y afectación de ecosistemas urbanos tales como cañadas, quebradas y ríos, que se convierten en botaderos de basuras y demás comportamientos infames en contra del medio ambiente que ha asumido el ser humano; estos actos de inconsciencia hacia la preservación del entorno natural y la ausencia de cultura ambiental ha motivado a nivel mundial, la búsqueda de un sin número de soluciones a estas problemáticas, entre las 
que se destacan la creación de zonas especiales de manejo ambiental, encaminados a la protección y recuperación de los bienes naturales que poseemos los habitantes del planeta. Este trabajo se concentra en la presentación de una alternativa para la rehabilitación productiva y el manejo sostenible de microcuencas urbanas teniendo como muestra una quebrada urbana del área metropolitana de Bucaramanga. El problema radica acerca del desaprovechamiento del terreno de las rondas de las microcuencas urbanas por la presencia de agentes contaminantes generados por los habitantes de cada sector y el desconocimiento de la comunidad del valor tan importante de este recurso natural. Esta investigación contiene las características más relevantes de una microcuenca urbana como lo son el estado de los recursos naturales tales como agua, suelo, fauna y flora; y cómo a partir de estos, es posible plantear una solución viable a la problemática del sector y brindar un valor agregado no solo ambientalmente, sino económica y socialmente, que permita el desarrollo sostenible de la comunidad.

\section{METODOLOGÍA}

Cuantitativa con un enfoque cuasi-experimental ideal para la caracterización ambiental de sectores naturales afectados por la intervención antrópica en deterioro del ambiente. Esta investigación incluye fases como la recolección de datos por medio de visitas, análisis social y de registros fotográficos; una segunda etapa con análisis de muestras e información recolectada, tercera etapa la caracterización de residuos sólidos, biológica, geológica y fisicoquímica de sus aguas para lograr identificar el daño ocasionado durante varios años; acompañada finalmente de la identificación de los problemas ambientales más críticos con el fin de establecer exactamente patrones de comportamiento ambiental en la quebrada.

Delimitación del área de estudio: La quebrada la Calavera es una corriente de bajo caudal desde su nacimiento en el barrio Altos de Bellavista hasta su unión con la Quebrada Zapamanga y abarca los barrios Panorama, Escoflor, Adeviposan, Bellavista, Santana, Villa Real sur, Guanatá, Lagos I, Rosales y Ciudad Valencia, con las coordenadas $7^{\circ} 04^{\prime} 30.60^{\prime \prime} \mathrm{N}$ y $73^{\circ} 05^{\prime} 57.46^{\prime \prime}$. O a una altura media de $891 \mathrm{~m}$.

\section{RESULTADOS Y DISCUSIÓN}

\subsection{Identidad Social}

Mediante la aplicación de modelos para proyectos comunitarios se genera una experiencia piloto para recuperación de cañadas o microcuencas urbanas mediante la Individualización, interacción, institucionalización, colectividad y gobernanza en la microcuenca la Calavera de Floridablanca se generan evidencias como el comité ambiental para la recuperación de la quebrada gestiona el desarrollo de planes, programas, proyectos e iniciativas populares legislativas y normativas ante las corporaciones públicas [1].

\subsection{Análisis de Residuos}

En el recorrido realizado a la cañada de la quebrada la Calavera, se encontraron problemas de contaminación que se presentan con mayor frecuencia en el área metropolitana de Bucaramanga, los que se lograron evidenciaren la cañada son; vertimientos de aguas residuales domesticas de manera directa por establecimientos ilegales que no cuentan con servicio de alcantarillado y vertimientos por conexiones erradas en el sistema de alcantarillado y taponamientos en el sistema de aliviaderos en los colectores combinados; botaderos de escombros y basuras al cauce de la quebrada, en algunos sectores la corriente es utilizada como sumideros; criadero de animales, invasiones y ocupación y perdida de las márgenes de protección de la quebrada. Se toma un trayecto de $500 \mathrm{~m}$ y se usa el método del cuarteo lo que se ha caracterizado denota la alta contaminación antrópica: plástico $4,36 \%$, papel $0,176 \%$; Vidrio $0,97 \%$; cartón $5,4 \%$; materia orgánica $3,53 \%$; respel $1,06 \%$; metales $2,51 \%$; escombros de construcción $62,09 \%$; icopor u otros $19,9 \%$.

\subsection{Calculo de $\mathrm{CO}_{2}$ método no destructivo}

Durante la investigación se plantea determinar la relación biomasa/ absorción de $\mathrm{CO}_{2}$ como estudio piloto para microcuencas urbanas como posibles sumideros de dióxido de carbono dando respuesta así a un problema puntual de la comunidad al no presentarse estudios anteriores de este tipo, el área en estudio fue de $300 \mathrm{~m}$.

Se aplica este método al contar con todos los datos necesarios (Diámetro de altura de pecho-DAP-, altura total y densidad básica de la madera) para aplicar la ecuación de estimación de biomasa más exacta descrita por Brown et al. (1989) $[2,3,5]$ de tipo exponencial y derivada para el bosque húmedo. El área de estudio fue de $300 \mathrm{~m}^{2}$ en la microcuenca:

$$
\text { Bte } \quad\left(-2,4090+0,9522 \ln \left(\mathrm{d}^{\wedge} 2 \mathrm{~h} \delta\right)\right)
$$

En las Tablas 1 y 2 se muestran parte de los resultados obtenidos y se registran los datos totales del área en estudio, con lo cual es de notar la importancia de una microcuenca como sumidero o absorción de dióxido de carbono. La cantidad de carbono almacenado en los árboles depende de las especies, las condiciones ambientales de crecimiento, la edad del árbol (ver ecuación 2) y la densidad de los árboles circundantes. 
Tabla 1. Determinación de Co2 en parte de la masa arbórea de la ronda microcuenca la calavera.

\begin{tabular}{|c|c|c|c|c|c|}
\hline $\begin{array}{c}\text { Nombre } \\
\text { común }\end{array}$ & $\begin{array}{c}\text { HT (altura } \\
\text { total-m) }\end{array}$ & $\begin{array}{c}\text { DAP (diámetro } \\
\text { altura de pecho- } \\
\mathbf{c m}\end{array}$ & $\begin{array}{c}\text { Biomasa total= Bt } \\
(\mathbf{k g})\end{array}$ & $\begin{array}{c}\text { Biomasa } \\
\text { total } \\
\text { (ton) }\end{array}$ & $\begin{array}{c}\text { Carbono } \\
\text { (ton) }\end{array}$ \\
\hline Oiti & 10,8 & 57,2956455 & 1356,843019 & 1,356843019 & 0,597010928 \\
\hline Gallinero & 7,6 & 15,2788388 & 78,34820675 & 0,078348207 & 0,034473211 \\
\hline Gallinero & 8,2 & 11,4591291 & 48,69883573 & 0,048698836 & 0,021427488 \\
\hline Limon & 13,8 & 20,0534759 & 232,0702949 & 0,232070295 & 0,10211093 \\
\hline Ceiba & 11,2 & 34,0590782 & 521,65865 & 0,52165865 & 0,229529806 \\
\hline Mango & 11 & 56,3407181 & 1337,263371 & 1,337263371 & 0,588395883 \\
\hline Pata vaca & 8,8 & 27,3745862 & 273,5002935 & 0,273500293 & 0,120340129 \\
\hline
\end{tabular}

Fuente. Elaboración propia.

En la Tabla 2 se presentan los resultados obtenidos de carbono y dióxido de carbono para el estudio; que se obtienen a partir de valores porcentuales ya estandarizados como se manifiesta.

Tabla 2. Biomasa total microcuenca la calavera.

\begin{tabular}{|l|l|}
\hline Biomasa Total $(\mathrm{Kg})$ & 7148,866992 \\
\hline Biomasa Total (Ton) & 7,148866992 \\
\hline Carbono (Kg) & 3145,501476 \\
\hline Carbono (Ton) & 3,145501476 \\
\hline Dióxido de Carbono (Kg) & 11543,99042 \\
\hline Dióxido de Carbono (Ton) & 11,54399042 \\
\hline
\end{tabular}

Fuente. Elaboración propia.

La masa arbórea encontrada es diversa, pero por su altura muy baja y diámetro menor a $40 \mathrm{~cm}$ en la mayoría de las especies no son incluidas debido a la ecuación empleada.

Para el propósito de esta investigación, las ecuaciones se utilizan para obtener una estimación promedio de vida de los arboles muestreados y excluyen al carbono en el suelo. Teniendo en cuenta que el $35 \%$ del peso de un árbol es agua y el peso seco $\left(\mathrm{a} 70^{\circ} \mathrm{C}\right)$ equivalente al $50 \%$ de un árbol es carbono [2] Además que el $20 \%$ de la biomasa de árboles es subterránea en las raíces se ha usado un factor de $120 \%$ (ver ecuación 1). Para determinar la cantidad equivalente de dióxido de carbono, se multiplica por un factor de 3,67 como se muestra en la ecuación 2.
(2)

$\mathrm{CO}_{2}$ absorbido en cada árbol $(\mathrm{kg})=$ Masa Arbórea $(\mathrm{kg}$ de biomasa fresca) x $65 \%$ (peso seco) x 50\% (\% de carbón) $\mathrm{x} 3.67 \times 120 \%=\mathrm{X}$

$\mathrm{CO}_{2}$ absorbido por árbol por año $(\mathrm{kg})=\mathrm{X} / \mathrm{edad}$ del árbol (años)

\subsection{Análisis Respirométrico}

Las mediciones se realizaron luego de 24 horas de tomada la muestra de $100 \mathrm{~g}$ de suelo para iniciar la valoración. Se libera el $\mathrm{CO}_{2}$ con cloruro de Bario al $1 \%$ y posteriormente aforamos a $100 \mathrm{~mL}$ usando agua destilada. Para calcular el dióxido de carbono se aplica la siguiente fórmula:

$\mathrm{mg}$ de $\mathrm{CO}_{2}$ producido $=(($ mililitro de hidróxido $\mathrm{x}$ Normalidad del hidróxido) - (mililitro de ácido $\mathrm{x}$ normalidad del ácido)) x 22

Nota: Se usa un factor de conversión de 22 para calcular la cantidad, en miligramos, de $\mathrm{CO}_{2}$ absorbido por el captador de $\mathrm{NaOH}$.

A pesar del alto grado de intervención y contaminación evidenciadas en la visita realizada a la quebrada La Calavera, se aprecia que existe un elevado potencial de autodepuración de la quebrada, en razón a la existencia del alta pendiente de la microcuenca y la naturalidad que todavía presenta el lecho de la quebrada. 
Tabla 3. Dióxido de carbono en muestras de suelo de la quebrada la calavera

\begin{tabular}{|c|c|c|c|c|}
\hline $\begin{array}{c}\text { Prueba 1 } \\
\text { respiración del } \\
\text { suelo }\end{array}$ & $\begin{array}{c}\text { Prueba 2 mg } \\
\text { respiración del } \\
\text { suelo }\end{array}$ & $\begin{array}{c}\text { Prueba 3 } \\
\text { respiración del } \\
\text { suelo }\end{array}$ & $\begin{array}{c}\text { Prueba } \\
\text { respiración del } \\
\text { suelo }\end{array}$ & $\begin{array}{c}\text { Prueba 5 } \\
\text { respiración del } \\
\text { suelo }\end{array}$ \\
\hline $\mathrm{kg} \mathrm{C}\left(\mathrm{en} \mathrm{CO}_{2}\right) / \mathrm{ha} / \mathrm{d}$ & $\mathrm{kg} \mathrm{C}\left(\mathrm{en} \mathrm{CO}_{2}\right) / \mathrm{ha} / \mathrm{d}$ & $\mathrm{kg} \mathrm{C}\left(\mathrm{en} \mathrm{CO}_{2}\right) / \mathrm{ha} / \mathrm{d}$ & $\mathrm{kg} \mathrm{C}\left(\mathrm{en} \mathrm{CO}_{2}\right) / \mathrm{ha} / \mathrm{d}$ & $\mathrm{kg} \mathrm{C}\left(\mathrm{en} \mathrm{CO}_{2}\right) / \mathrm{ha} / \mathrm{d}$ \\
\hline 37,86 & 36,73 & 36,82 & 38,88 & 39,92 \\
\hline 38,01 & 35,63 & 36 & 37,99 & 41,64 \\
\hline 37,01 & 35,57 & 35,88 & 38,67 & 39,62 \\
\hline
\end{tabular}

Fuente. Elaboración propia.

Sin embargo, se requiere que sean retirados los vertimientos que actualmente llegan a la quebrada.

Tabla 4. Listado de especies encontradas en la quebrada La Calavera.

\begin{tabular}{|c|c|}
\hline Balso & Ochromalagopus \\
\hline Caracolí & Anacardiumexcelsum \\
\hline Cordoncillo & Pipersp \\
\hline Corozo & Bactrisminor \\
\hline Gaque & Clusia multiflora \\
\hline Guamo & Inga sp. \\
\hline Guayabo & Psidiumguajava \\
\hline Heliconia & Heliconia stricta \\
\hline Manchador & Vismiabacifera \\
\hline Mosquero & Crotonferruginea \\
\hline Yarumo & Cecropiapeltata \\
\hline
\end{tabular}

Fuente. Elaboración propia.

Es de resaltar que en algunos tramos de la quebrada no se respeta la zona de ronda y por el contrario se evidencia la presencia de construcciones a una poca distancia del margen de la quebrada, con un deterioro evidente del entorno natural y un alto riesgo de daño a dichas construcciones, particularmente durante eventos extremos de incremento del caudal de la quebrada. Igualmente, se observa la presencia de basuras en gran parte del cauce de la quebrada, así como la inadecuada disposición de medicamentos vencidos, los cuales se convierten en un elemento de alto riesgo toxicológico, particularmente para las personas que entren en contacto con el agua de la quebrada. En la Tabla 4, se enumeran algunos de las especies vegetales presentes en los márgenes de la quebrada.

Con lo descrito en la Tabla 3 el suelo se encuentra en un estado ideal de actividad biológica y posee adecuada materia orgánica y activas poblaciones de microorganismos ya que se encuentra en el rango $35.84-$ $71.68 \mathrm{Kg}$ de C [5].

Tabla 5. Familias de macroinvertebrados acuático.

\begin{tabular}{|c|c|c|}
\hline Gastropoda & Mesograstropoda & Hidrobiidae \\
\hline Gastropoda & Basommatophora & Physidae \\
\hline Hirudinea & Rhynchobdellida & Glossiphoniidae \\
\hline Turbelaria & Tricladida & Planariidae \\
\hline Insecta & Diptera & Quironomidae \\
\hline Insecta & Diptera & Muscidae \\
\hline Gastropoda & Mesograstropoda & Hidrobiidae \\
\hline Gastropoda & Basommatophora & Physidae \\
\hline
\end{tabular}

Fuente. Elaboración propia.

\subsection{Caracterización Biológica}

Se aprecia un alto grado de intervención y en algunos tramos las edificaciones se extienden hasta casi el margen de la quebrada. Por su parte la fauna de macroinvertebrados bentónicos muestra que prácticamente la totalidad de los organismos son bioindicadores de aguas con alto grado de contaminación por materia orgánica.

\subsection{Vegetación y cobertura del suelo}

La vegetación de los márgenes de la quebrada La Calavera corresponde esencialmente a una vegetación secundaria, caracterizada por un alto grado de intervención antrópica. Al implementar la metodología CorineLandCover [6], para identificar las coberturas del suelo en la zona de influencia de la quebrada La Calavera se observa la presencia de las coberturas: tejido urbano continuo y bosque de galería y ripário. 
Tabla 6. Clasificación granulométrica promedio trayecto escogido.

\begin{tabular}{|c|c|c|c|c|c|}
\hline GRAVA & 17,1 & $\%$ & $\mathrm{D} 10$ & 0,03 & $\mathrm{~mm}$ \\
\hline ARENA & 55,8 & $\%$ & $\mathrm{D} 30$ & 1,33 & $\mathrm{~mm}$ \\
\hline FINOS & 27,1 & $\%$ & $\mathrm{D} 60$ & 1,46 & $\mathrm{~mm}$ \\
\hline Cc & 46,7 & & $\mathrm{Cu}$ & 56,55 & \\
\cline { 1 - 3 } CLASIFICACION: & & & \multicolumn{4}{|c|}{ ARENAS LIMOSAS, } \\
MEZCLA DE ARENA Y \\
LIMO. (SM)
\end{tabular}

Fuente. Elaboración propia.

\subsection{Fauna asociada}

El muestreo de fauna se concentró en insectos y macroinvertebrados acuáticos, aunque durante el recorrido se pudieron identificar otras especies terrestres como hormigas del genero Atta $s p$. La tabla 5, muestra algunos de los insectos y macroinvertebrados encontrados en la quebrada La Calavera.

\subsection{Caracterización del Suelo}

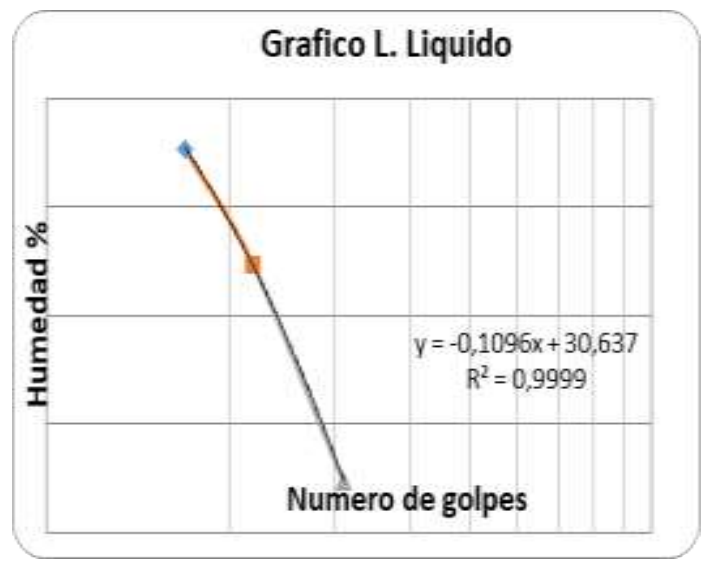

Figura 1. Índice de plasticidad del suelo. Fuente. Elaboración propia.

Tabla 7. Resultados índices plástico, sitios 1-2-3.

\begin{tabular}{|c|c|c|c|}
\hline sito & $\begin{array}{c}\text { Limite } \\
\text { Liquido }\end{array}$ & $\begin{array}{c}\text { Limite } \\
\text { Plástico }\end{array}$ & $\begin{array}{c}\text { Índice } \\
\text { Plástico }\end{array}$ \\
\hline 1 & N.L.L. & N.L.P. & N.I.P. \\
\hline 2 & 28,24 & 24,74 & 3,50 \\
\hline 3 & 27,90 & 19,87 & 8,02 \\
\hline
\end{tabular}

Fuente. Elaboración propia.
Se estudian sectores que aportan tipos representativos de suelo a lo largo del recorrido de la quebrada, y así poder conocer la tipología del suelo y sus posibles afectaciones por la acción antrópica a lo largo de todos estos años. Se realiza la entrada al terreno desde el afloramiento de la cañada hasta su desembocadura en la quebrada Zapamanga.

\subsection{Ensayo granulometría}

Los análisis han sido el estudio de suelos, identificación de material litográfico, fauna y flora respectivamente. El terreno de muestreo es arenoso en la mayoría de su trayecto, lo cual se verifica con los resultados de la tabla 6.

\subsubsection{Resultado ensayo textura del suelo.}

Mediante el tacto y la apariencia de la muestra se puede determinar aproximadamente la textura del suelo. Humedeciéndola se forman cilindros y esferas y compararlas entre sí. La muestra presenta una apariencia color café, pegajoso, blando y brillante. El Suelo es muy suave al tacto. Formas bolas y rollos bien definidos y de superficie lisa. Cuando se seca es resistente a desmoronarse. (Arcillo-Limosa) - (Ar L).

\subsubsection{Resultados ensayo Límites de Atterberg del suelo.}

El suelo objeto de estudio por sus propiedades de origen aluvial, presenta una característica muy importante que distingue este tipo de suelos (ligeramente plásticos), que se evidencia en la tabla 7 y figura 1, donde se observa el comportamiento inversamente proporcional entre la permeabilidad del suelo y el índice plástico; debido a la composición del suelo, en la muestra, tomada en la parte baja de la cuenca, el suelo es en su mayoría areno limoso, que en comparación con los otros dos suelos cuenca arriba, es nulo su índice plástico, característico de suelos limosos, que son de difícil manejo para cimentaciones, dado a que tienden a ser inestables. Esa expansión y contracción genera daños progresivos en los suelos, reflejados fisuras, grietas y giros en muros y elementos estructurales, a causa de movimientos desiguales de sus cimientos. Lo que en particular inquieta por el contenido de arcillas elevado (63\% Finos).

\subsection{Análisis Hídrico}

Esta "caracterización" e identificación de vertimientos (ver Tabla 8 y 10) analiza las principales características 
Tabla 8. Puntos críticos de la quebrada La Calavera.

\begin{tabular}{|c|c|c|}
\hline GEOREFERENCIACIÓN & OBSERVACIONES & $\begin{array}{l}\text { REGISTRO } \\
\text { FOTOGRAFICO } \\
\end{array}$ \\
\hline $\begin{array}{l}\text { N: } 1274018 \\
\text { E: } 1107386 \\
\text { H: } 857 \mathrm{~m}\end{array}$ & $\begin{array}{l}\text { Al momento de la visita se } \\
\text { observó filtraciones y reboses } \\
\text { del Pozo de inspección del } \\
\text { sistema de alcantarillado }\end{array}$ & \\
\hline $\begin{array}{l}\mathrm{N}: 1274391 \\
\mathrm{E}: 1107920 \\
\mathrm{H}: 895 \mathrm{~m}\end{array}$ & $\begin{array}{l}\text { Conexión errada, durante el } \\
\text { recorrido se percibieron olores } \\
\text { molestos y cambio en las } \\
\text { características organolépticas } \\
\text { del recurso hídrico. }\end{array}$ & \\
\hline $\begin{array}{l}\text { N: } 1274005 \\
\text { E: } 1107386 \\
\text { H: } 857 \mathrm{~m}\end{array}$ & $\begin{array}{l}\text { Posibles conexiones erradas } \\
\text { antes de la confluencia de la } \\
\text { Quebrada la Calavera con la } \\
\text { Quebrada La Zapamanga. }\end{array}$ & \\
\hline $\begin{array}{l}\text { N: } 1274178 \\
\text { E: } 1107924 \\
\text { H: } 881 \mathrm{~m}\end{array}$ & $\begin{array}{l}\text { Debajo del puente que } \\
\text { comunica los Barrios Guanata } \\
\text { con Lagos I se presentaban } \\
\text { vertimientos de aguas } \\
\text { residuales domésticas, se } \\
\text { percibieron olores molestos y } \\
\text { cambios en la calidad del } \\
\text { recurso hídrico de la Quebrada } \\
\text { la Calavera. }\end{array}$ & \\
\hline
\end{tabular}

Fuente. Elaboración propia.

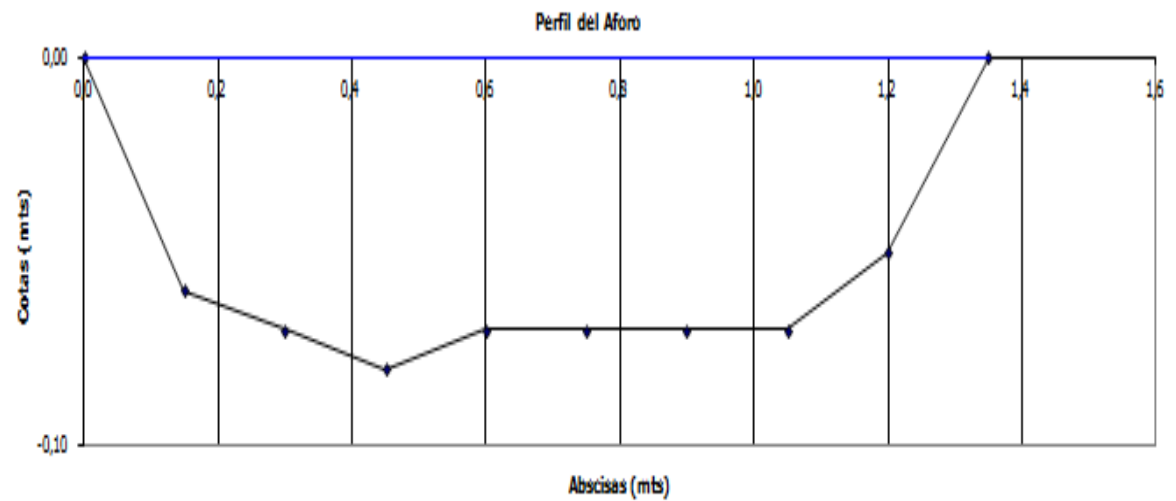

Figura 2. Perfilde la quebrada La Clavera. Fuente. Elaboración propia. 
Fisicoquímicas del recurso hídrico en la quebrada la Calavera. En este estudio de carácter principalmente académico los límites de la calidad del agua se expresan en relación con el tipo de uso que se le da al agua. Se aplicaron los límites expresados en el Decreto 1594 de 1984 del Ministerio de Agricultura.

\subsection{Diagnóstico Organoléptico: En campo}

Se miden estos parámetros en un tramo de $1000 \mathrm{~m}$ de longitud de la quebrada. Se realizaron observaciones como tipo de sustrato, apariencia, color y olor. Los datos fueron registrados en tablas y gráficos, con el fin de establecer las diferencias a lo largo de las quebradas. Se realiza el análisis organoléptico (ver tabla 8) a parte de la quebrada desde jurisdicción de la Iglesia San Agustín hasta su unión con la quebrada Zapamanga para identificar los sectores que tienen distinta litología, morfología y diversidad en fauna y flora para elaborar una ruta que involucre todos los componentes biogeoquímicos.

Tabla. 9. Resultados organolépticos microcuenca la calavera.

\begin{tabular}{|l|l|}
\hline \multirow{2}{*}{ APARIENCIA } & $\begin{array}{l}\text { Ligera cantidad de sedimentos en } \\
\text { partes de la quebrada. Poca } \\
\text { profundidad en la mayoría del } \\
\text { recorrido (menos de 1 metro). }\end{array}$ \\
\hline COLOR & $\begin{array}{l}\text { Incolora y traslucida en la mayoría } \\
\text { del recorrido. } \\
\text { En algunos sectores el agua presenta } \\
\text { baja profundidad y alta turbidez. }\end{array}$ \\
\hline OLOR & $\begin{array}{l}\text { Inodoro y fresco en la mayoría del } \\
\text { trayecto. } \\
\text { En algunos sitos se percibe un ligero } \\
\text { aroma a gallinaza. }\end{array}$ \\
\hline SABOR & $\begin{array}{l}\text { No aplica, por posible carga } \\
\text { contaminante perjudicial para la } \\
\text { salud. }\end{array}$ \\
\hline TEXTURA & \begin{tabular}{l} 
Franco Arenosa en todo el terreno. \\
\hline
\end{tabular} \\
\hline
\end{tabular}

Fuente. Elaboración propia.

Nota: En todo el recorrido se encontraron cantidades recurrentes de residuos sólidos domiciliarios en el agua y sus terrenos adyacentes que por escorrentía son agentes potencialmente contaminantes de la quebrada.
Tabla 10. Promedio de análisis fisicoquímico de la quebrada La Calavera.

\begin{tabular}{|c|c|c|}
\hline PARÁMETRO & RESULTADO & UNIDADES \\
\hline $\begin{array}{l}\text { Temperatura } \\
\text { Ambiente }\end{array}$ & 27,2 & ${ }^{\circ} \mathrm{C}$ \\
\hline $\begin{array}{l}\text { Temperatura } \\
\text { del Agua }\end{array}$ & 23,3 & ${ }^{\circ} \mathrm{C}$ \\
\hline pH & 8,30 & Unid de $\mathrm{pH}$ \\
\hline OD & 7,30 & $\mathrm{mg} \mathrm{O}_{2} / \mathrm{L}$ \\
\hline Conductividad & 219 & $\mu \mathrm{S} / \mathrm{cm}$ \\
\hline Turbidez & 18,6 & $\mathrm{NTU}$ \\
\hline DQO & 21,1 & $\mathrm{mg} \mathrm{O}_{2} / \mathrm{L}$ \\
\hline $\mathrm{DBO}_{5}$ & 3,67 & $\mathrm{mg} \mathrm{DBO}_{5} / \mathrm{L}$ \\
\hline $\begin{array}{l}\text { Nitrógeno } \\
\text { Total Kjeldahl }\end{array}$ & 2,98 & $\mathrm{mg} \mathrm{N} / \mathrm{L}$ \\
\hline Nitritos & 0,139 & $\begin{array}{ll}\mathrm{mg} & \mathrm{NO}_{2}^{-}- \\
\mathrm{N} / \mathrm{L} & \\
\end{array}$ \\
\hline Nitratos & 1,16 & $\underset{\mathrm{N} / \mathrm{L}}{\mathrm{mg}} \mathrm{NO}_{3}^{-}-$ \\
\hline Fosforo Total & 0,31 & $\mathrm{mg} \mathrm{P} / \mathrm{L}$ \\
\hline Sólidos totales & 190 & $\mathrm{mg} / \mathrm{L}$ \\
\hline $\begin{array}{l}\text { Sólidos } \\
\text { Suspendidos }\end{array}$ & 19,5 & $\mathrm{mg} / \mathrm{L}$ \\
\hline $\begin{array}{ll}\text { Grasas } & \mathbf{y} \\
\text { aceites } & \end{array}$ & $<5,0$ & $\mathrm{mg} / \mathrm{L}$ \\
\hline $\begin{array}{l}\text { Coliformes } \\
\text { Totales }\end{array}$ & $7,0 \times 10^{4}$ & $\begin{array}{l}\mathrm{NMP} / 100 \\
\mathrm{~mL}\end{array}$ \\
\hline $\begin{array}{l}\text { Coliformes } \\
\text { Fecales }\end{array}$ & $2,2 \times 10^{4}$ & $\begin{array}{l}\text { NMP/100 } \\
\mathrm{mL}\end{array}$ \\
\hline $\begin{array}{l}\text { Sólidos } \\
\text { volátiles }\end{array}$ & 0,6 & $\mathrm{mg} / \mathrm{dm}^{3}$ \\
\hline
\end{tabular}

Fuente. Elaboración propia.

Los perfiles de la quebrada pueden presentan pendientes medias a altas (entre 50-80 \%); Esta característica hace que estas aguas sean rápidas y que las quebradas tengan un cauce elevado sin mucha acumulación de material finamente particulado.

En comparación a lo estipulado en el artículo 39 del decreto 1594 de 1984, el cual establece los criterios de calidad admisibles para la destinación del recurso agua para consumo humano, los valores de turbiedad [7] (tabla 9) oscilan dentro del rango del límite permisible, pues lo estipulado es 10 NTU y la quebrada presenta una turbidez 
mínima de 4,2 y 9,22 NTU con un valor máximo de 18,6 NTU que en el caso de ser utilizada para consumo humano, con un proceso de tratamiento previo se puede corregir. A mayor valor de DBO, mayor es la contaminación del agua. El rango de valores está en mínimo 3,7 y máximo 20, siendo valores bajos de carga orgánica que permiten mediante un tratamiento previo hacer uso del agua de la quebrada. En la Demanda Química de Oxigeno se utilizan agentes oxidantes en un medio ácido para efectuar la oxidación de la materia orgánica. Tal como la DBO, a mayor valor, mayor es la contaminación presente en el agua. El valor mínimo es $21,1 \mathrm{mg} \mathrm{O}_{2} / \mathrm{L}$ y máximo $53,6 \mathrm{mg} \mathrm{O}_{2} / \mathrm{L}$, valores son bajos que permiten la utilización del agua para consumo humano previo a un sistema de tratamiento.

\section{CONCLUSIONES}

La disposición de escombros en la quebrada la Calavera no se da en la mayoría de los casos o puntos de muestreo directamente en el cauce, pero muchos de los materiales se desplazan a éste por la acción, el viento y escorrentía, aumentando los sólidos [8]. También relevantes en la corriente los materiales de tipo flotante, que son trasportados a través del lecho y que tienen bajos niveles de biodegradabilidad. La afectación del recurso está relacionada con el deterioro del paisaje y la ocurrencia de impactos negativos desde el punto de vista visual, además de la estrecha relación que tienen con la ocurrencia de taponamientos del cauce desbordamientos e inundaciones [9].

Con el modelo social que se realizó se ponen de manifiesto planes y acciones de inmediato cumplimiento en las instituciones integrales contando así a la comunidad, autoridades locales y academia.

Se determina que la quebrada la Calavera tiene un alto grado de intervención antrópica que afecta severamente la fauna y flora de su entorno. Evidencia de dicho grado de intervención son los vertimientos que caen a la quebrada provenientes del sistema de alcantarillado del municipio de Floridablanca, algunas edificaciones que se extienden hasta casi el lecho de la quebrada y la remoción de casi la totalidad de la vegetación primaria que constituía el bosque ripário de los márgenes de la quebrada.

La fauna acuática de la quebrada es en su gran mayoría indicadora de aguas con alto grado de contaminación y se caracteriza por elementos faunísticos tolerantes a la contaminación, mientras que las especies indicadoras de aguas con buena calidad han desaparecido en su totalidad. A pesar del grado de intervención observado en la quebrada La Calavera, se observa que el potencial de autodepuración de la misma es alto, debido al alto grado de pendiente de la microcuenca y a la naturalidad que todavía presenta el lecho de la quebrada.

Como análisis final y reconociendo que una de las características mecánicas del suelo más importante es la compactación o densidad del suelo, la quebrada la calavera como se muestro el suelo con mejores condiciones para soportar carga cuenta sitios que son de textura arenosa lo que dificulta entre otras cosas la cohesión de las partículas del terreno dado que son suelos granulares no cohesivos, y más aún con la particularidad de contener arenas sueltas de comportamiento esponjoso, con baja resistencia al corte directo y de manejo especial en cimentaciones y taludes.

Es recomendable caracterizar en cada microcuenca, la geomorfología, tipo y usos de suelos y cobertura vegetal, acompañado del inventario completo de las corrientes superficiales de agua, así como de afloramientos de aguas subterráneas [10].

\section{AGRADECIMIENTOS}

Los autores expresan su agradecimiento a la Universidad de Santander por el préstamo de sus laboratorios para llevar a cabo esta investigación.

\section{REFERENCIAS}

[1] N. E. González y M. C. Moreno. Modelo de intervención para servicios de consultoría social en ingeniería. Revista ESAICA, Vol.1 n ${ }^{\circ}$, pp. 70-75, junio 2015.

[2] Jiménez P., J. Fijación de carbono en la Reserva de la Biosfera "El Cielo". Informe Técnico Final. Facultad de Ciencias Forestales. UANL. México. 2005.

[3] Cuatrecasas, J. Aspectos sobre la vegetación natural de Colombia. Rev. Academia Colombiana de Ciencias Exactas Físicas y naturales vol.10.1958.

[4] Holdridge, L. Ecología basada en zona de vida. Trad. del inglés por Jiménez, H. Segunda reimpresión. Instituto Interamericano de Cooperación para la Agricultura. San José, Costa Rica. 216 p. 1982.

[5] Holdridge, L. R. Life Zone Ecology.Tropical Science Center. San José, Costa Rica. 1967.

[6] IDEAM. Leyenda Nacional de Coberturas de la Tierra. Metodología CORINE LandCover adaptada para Colombia Escala 1:100.000. Instituto de Hidrología, Meteorología y Estudios Ambientales. Bogotá, D. C., 72p. 2010. 
[7] Ministerio de Agricultura de Colombia. Decreto 1594 de 1984. Bogotá, D.C.

[8] Woods End Research. Guide to solvita testing and managing your soil. Woods End Research Laboratory, Inc., Mt. Vernon, ME. 1997.

[9] Brown, Sandra. Lugo, Ariel. "Estimación de biomasa superficial en bosques tropicales húmedos del Amazonas en Brasil”. En: Interciencia. Vol. n 17, 1 Ene -Feb, 1992, Caracas: redalyc,, 9 páginas. ISSN: 0378-1844. 1992.

[10] Guzmán, Jorge et al. Modelos hidrológicos en la cuenca experimental de rio sucio, municipio de Tona, Santander. Revista UIS Ingenierías, [S.1.], v. 7, n. 1, feb. 2010. ISSN 2145-8456. [En Línea] Disponible en: $<$ http://revistas.uis.edu.co/index.php/revistauisingenieri as/article/view/392>. 